Proyecciones Journal of Mathematics

Vol. 29, No 2, pp. 93-100, August 2010.

Universidad Católica del Norte

Antofagasta - Chile

\title{
ON TURÁN'S TYPE INEQUALITIES FOR SOME SPECIAL FUNCTIONS AND OPERATORS
}

\author{
HARI SINGH PARIHAR \\ POORNIMA INSTITUTE OF ENG. AND TECH., INDIA \\ Received : September 2009. Accepted: April 2010
}

\begin{abstract}
The purpose of this paper is to use the generalized Schwarz inequality to give a short proofs of new Turán-type inequalities for some special functions and operators.

Subjclass [2000] : Primary 26A33, Secondary 30C45.

Keywords : Turanians, Negapolygamma function, Generalized Hurwitz- Lerch Zeta function, Mellin transforms, unified Riemann zeta function, Riemann-Liouville fractional integral operator.
\end{abstract}




\section{Introduction}

P. Turán showed in a 1946 letter to Szegö that

$$
\Delta_{n}(x)=P_{n}^{2}(x)-P_{n-1}(x) P_{n+1}(x) \geq 0, \quad x \in[-1,1], n \geq 1
$$

where $P_{n}(x)$ denotes the n-th Legendre polynomial. Szegö [6] gave four non trivial proofs. This classical result has been extended in several directions: Bessel function of the first kind, modified Bessel functions, Hermite polynomials, Laguerre polynomials, ultraspherical polynomials etc. In view of the interest in inequalities, Karlin and Szegö named the determinants, such as (1.2)Turánians.

$$
\left|\begin{array}{cc}
P_{n}(x) & P_{n+1}(x) \\
P_{n+1}(x) & P_{n+2}(x)
\end{array}\right| \leq 0, \quad-1 \leq x \leq 1
$$

where $n=0,1,2, \ldots$ and equality occurs only if $x= \pm 1$.

The proof given by Turán is based on the recurrence relation [5, p. 81],

$$
\left\{\begin{array}{cc}
(n+1) P_{n+1}(x)=(2 n+1) x P_{n}(x)-n P_{n-1}(x) & \\
P_{-1}(x)=0, P_{0}(x)=1, & n=1,2, \ldots
\end{array}\right.
$$

and on the differential relation $[5, \mathrm{p} .83]$

$$
\left(1-x^{2}\right) P_{n}^{\prime}(x)=n P_{n-1}(x)-n x P_{n}(x)
$$

L. Lorch [4] derived Turán-type inequalities for the positive zeros $C_{\nu K}$, $K=1,2, \ldots$ of the general Bessel function

$$
C_{\nu}(x)=J_{\nu}(x) \cos \theta-Y_{\nu}(x) \sin \theta, 0 \leq \theta<\pi
$$

where $J_{\nu}(x)$ and $Y_{\nu}(x)$ denote the Bessel function of the first and the second kind respectively. 
Finally, the corresponding results for the positive zeros $C_{\nu K}^{\prime} \nu \geq 0, \quad K=$ $1,2, \ldots$ of the derivative $C_{\nu}^{\prime}(x)=\frac{d}{d x}\left[C_{\nu}(x)\right]$ and for the zeros of Hermite Laguerre and ultraspherical polynomials have been established in [1] [2] and [3] respectively.

The object of this paper is to prove new Turán-type inequalities for some special functions like unified Riemann zeta function, Negapolygamma function, generalized Hurwitz-Lerch Zeta function and some operator like Mellin transform, Riemann-Liouville fractional integral operator.

The approach used in this paper is based, prevalently on Sturm theory and is different from the one used in the above mentioned papers. Here our main tool is the following generalized Schwarz inequality.

$$
\int_{a}^{b} g(t)[f(t)]^{m} d t \int_{a}^{b} g(t)[f(t)]^{n} d t \geq\left[\int_{a}^{b} g(t)[f(t)]^{\frac{m+n}{2}} d t\right]^{2}
$$

where $f$ and $g$ are two non negative functions of a real variable and $\mathrm{m}$ and $\mathrm{n}$ belonging to a set $R$ of real numbers, such that the integral in (1.5) exists.

\section{Main Results}

Theorem 2.1. The Legendre polynomial of order $n$ is denoted by $P_{n}(x)$ in [10]. Then

$$
P_{n-1}(x) P_{n+1}(x)-P_{n}^{2}(x) \geq 0,|x| \geq 1
$$

Proof. From [13, pp.203], we have

$$
P_{n}(\cosh \alpha)=\frac{1}{\pi} \int_{0}^{\infty}(\cosh \alpha+\sinh \alpha \cos t)^{n} d t
$$

and (1.5) with $n-1, n+1$ instead of $m, n$, to establish the inequality for $x \geq 1$, and then use the fact that $P_{n}(x)$ is ever for $n$ even, odd for $n$ odd, to prove it for $x \leq-1$.

This completes the proof.

Theorem 2.2. The unified Riemann zeta function is denoted by $\phi_{\mu}(z, s, a)$ in $[7, p .87]$. Then 


$$
(s+1) \frac{\phi_{\mu}(z, s, a)}{\phi_{\mu}(z, s+1, a)} \geq(s) \frac{\phi_{\mu}(z, s+1, a)}{\phi_{\mu}(z, s+2, a)}, s>1 .
$$

Proof. For $s>1$, the unified Riemann zeta function satisfies the integral relation

$$
\phi_{\mu}(z, s, a)=\frac{1}{\Gamma(s)} \int_{0}^{\infty} t^{s-1} \frac{e^{-a t}}{\left(1-z e^{-t}\right)^{\mu}} d t, \quad s>1
$$

By (1.5) with $g(t)=\frac{e^{-a t}}{\left(1-z e^{-t}\right)^{\mu}}, \quad f(t)=t$ and $a=0, \quad b=+\infty$.

We obtain

$$
\begin{gathered}
\int_{0}^{\infty} t^{s-1} \frac{e^{-a t}}{\left(1-z e^{-t}\right)^{\mu}} d t \int_{0}^{\infty} t^{s+1} \frac{e^{-a t}}{\left(1-z e^{-t}\right)^{\mu}} d t \geq \\
{\left[\int_{0}^{\infty} t^{s} \frac{e^{-a t}}{\left(1-z e^{-t}\right)^{\mu}} d t\right]^{2} .}
\end{gathered}
$$

Furthermore using (2.4) the inequality becomes

$$
\phi_{\mu}(z, s, a) \Gamma(s) \phi_{\mu}(z, s+2, a) \Gamma(s+2) \geq \phi_{\mu}^{2}(z, s, a) \Gamma^{2}(s+1)
$$

or by the functional relation $\Gamma(s+1)=s \Gamma(s)$,

$$
(s+1) \phi_{\mu}(z, s, a) \phi_{\mu}(z, s+2, a) \geq(s) \phi_{\mu}^{2}(z, s+1, a)
$$

This completes the proof.

Remark 2.1. In the above theorem(2.2), if we put $a=\mu=z=1$ then the given relation satisfies for the Riemann zeta function.

Theorem 2.3. We denote by $\psi^{(-n)}(z)$ the Negapolygamma function[9]. Then

$$
\text { (n) } \frac{\psi^{(-n)}(z)}{\psi^{(-n-1)}(z)} \geq(n-1) \frac{\psi^{(-n-1)}(z)}{\psi^{(-n-2)}(z)}, z>0
$$

and $n$ is an arbitrary positive integer. 
Proof. For $z>0$, the negapolygamma function can be written in integral form as

$$
\psi^{(-n)}(z)=\frac{1}{\Gamma(n-1)} \int_{0}^{z}(z-t)^{n-2} \log \Gamma(t) d t, \quad z>0
$$

when $n$ is an arbitrary positive integer.

We write the above integral in suitable form and similar steps used like Theorem(2.2)and using(1.5). We arrive at the required result.

Theorem 2.4. The Mellin transform of a function $f(x)$ is defined as

$$
M_{s}\{f(x)\}=M\{f(x) ; s\}=\int_{0}^{\infty} x^{s-1} f(x) d x, \quad x>0
$$

Then

$$
M_{s}\{f(x)\} M_{p}\{f(x)\} \geq\left[M_{\frac{s+p}{2}}\{f(x)\}\right]^{2}
$$

where $\frac{s+p}{2}$ is an integer.

Proof. In the theorem, we choose the integer $p$ and $s$ both even or odd in such a way that $\frac{p+s}{2}$ is an integer.

By (1.5), we get

$$
\int_{0}^{\infty} x^{s-1} f(x) d x \int_{0}^{\infty} x^{p-1} f(x) d x \geq\left[\int_{0}^{\infty} x^{\frac{p+s}{2}-1} f(x) d x\right]^{2}
$$

that is

$$
M_{s}\{f(x)\} M_{p}\{f(x)\} \geq\left[M_{\frac{s+p}{2}}\{f(x)\}\right]^{2}
$$

This completes the proof.

Theorem 2.5. The Riemann -Liouville fractional integral operator[8] of a function $f(x)$ is defined as

$$
{ }_{{ }_{c}} D_{t}^{-\nu} f(t)=\frac{1}{\Gamma(\nu)} \int_{c}^{t}(t-x)^{\nu-1} f(x) d x, \quad x>0
$$


Then

$$
\Gamma(\nu){ }_{c} D_{t}^{-\nu} f(t) \Gamma(\mu){ }_{c} D_{t}^{-\mu} f(t) \geq\left[\Gamma\left(\frac{\mu+\nu}{2}\right) ._{c} D_{t}^{-\left(\frac{\mu+\nu}{2}\right)} f(t)\right]^{2}
$$

where $\frac{\mu+\nu}{2}$ is an integer.

Proof. We use the similar lines as used in the above theorems and get the proof of this theorem.

The generalized Hurwitz-Lerch Zeta function $\phi_{\alpha, \beta ; \gamma}(z, s, a)$ is recently defined by Garg, Jain and Kalla in [12] as follows:

$$
\phi_{\alpha, \beta ; \gamma}(z, s, a)=\frac{1}{\Gamma s} \int_{0}^{\infty} t^{s-1} e^{-a t}{ }_{2} F_{1}\left(\alpha, \beta, ; \gamma ; z e^{-t}\right) d t
$$

where $(\operatorname{Re}(a)>0 ; \gamma \neq 0,-1,-2, \ldots, \operatorname{Re}(s)>0)$ and $|z|<1$ or $|z|=1$ with $\operatorname{Re}(\gamma-\alpha-\beta)>0$, where $d t_{2} F_{1}(\alpha, \beta, d t ; \gamma ; z)$ is the Gauss hypergeometric function defined in [10].

Theorem 2.6. The generalized Hurwitz-Lerch Zeta function is denoted by $\phi_{\alpha, \beta ; \gamma}(z, s, a)$ in [12]. Then

$$
(s+1) \frac{\phi_{\alpha, \beta ; \gamma}(z, s, a)}{\phi_{\alpha, \beta ; \gamma}(z, s+1, a)} \geq(s) \frac{\phi_{\alpha, \beta ; \gamma}(z, s+1, a)}{\phi_{\alpha, \beta ; \gamma}(z, s+2, a)}, \quad s>1 .
$$

Proof. We use the similar lines as used in the above theorems and get the proof of this theorem.

\section{Acknowledgment}

The author is thankful to worthy referee for his fruitful suggestions. 


\section{References}

[1] Á. ELBERT AND A. LAFORGIA : Monotonicity results on the zeros of generalized Laguerre polynomials, J. Approx Theory, 51(2), pp. 168$174,(1987)$.

[2] Á. ELBERT AND A. LAFORGIA : Some monotonicity properties for the zeros of ultraspherical polynomials, Acta Math. Hung., 48, pp. 155159, (1986).

[3] A. LAFORGIA : Sturm theory for certain class of Sturm-Liouville equations and Turánians and Wronskians for the zero of derivative of Bessel functions, Indag. Math., 3, pp. 295-301, (1982).

[4] L. LORCH : Turánians and Wronskians for the zeros of Bessel functions, SIAM J. MATH.Anal.,11, pp. 223-227, (1980).

[5] G. SZEGÖ : Orthogonal Polynomial 4th ed. Amer. Math. Soc., Colloquium Publications,23 Amer. Math. Soc. Providence, RI (1975).

[6] G. SZEGÖ : On an inequality of P. Turán concerning Lengendre polynomials,Bull Amer. Math. Soc., 54, pp. 401-405, (1948).

[7] S. P. GOYAL AND A. PARASHAR : Some results for the unified Riemann zeta function, Kyungpook mathematical journal,42(1), pp. 8794, (2002).

[8] K. S. MILLER AND B. ROSS : An introduction to the Fractional calculus and Fractional Differential equations, John wiley Sons, Inc. Newyork, (1993).

[9] V. ADAMCHIK : Polygamma function of negative order, Jour. Comp.Appl. Math., 100, pp. 191-199, (1998).

[10] E. D. RAINVILLE : Special functions, Chelsea Publ. Co. Bronx,Newyork, (1971).

[11] I. N. SNEDDON : The use of integral transforms, Mc-Graw Hill, NewYork., (1972).

[12] M. GARG, K. JAIN and S. L. KALLA : On Generalized Hurwitz-Lerch Zeta functions, Appl. and Appl. Math., 4(1), pp. 26-39, (2009). 
[13] N. M. TEMME : Special Functions: An introduction to the Classical Functions of mathematical Physics, Wiley Pub., (1996).

\section{Hari Singh Parihar}

Department of Mathematics,

Poornima Institute of Engineering and Technology,

Jaipur,

India

e-mail : harisingh.p@rediffmail.com 